\title{
Capturing the failure of a cohesive granular step
}

\author{
Lydie Staron $^{1 *}$, Anais Abramian ${ }^{1}$ and Pierre-Yves Lagrée ${ }^{1}$ \\ ${ }^{1}$ Institut d'Alembert - Université Paris Sorbonne, 75252 Paris, France
}

\begin{abstract}
A Contact Dynamics algorithm is applied to simulate the failure of 2-dimensional granular steps. Focussing on one single value for both the adhesive force threshold and the contact friction, we track the signature of the failure in the cumulative displacement of the grains. The introduction of a displacement threshold allows for the identification of the onset of a rapid failure and the formation of the corresponding shear band. The latter gives access to an estimation of the failure orientation.

The robustness of the measure thus performed for one single run is assessed by carrying out a series of 10 additional simulations, showing a fairly reproducible behaviour.
\end{abstract}

\section{Introduction}

Granular materials are complex. Even the simplest sort one may think of - for instance mono-disperse disks evolving in dry air- exhibits a surprising level of complexity. The exact moment when a granular slope starts avalanching, and the interval of values for the static angle of repose, are well known examples of the uncertainty of the mechanical behaviour of a simple granular packing [1]. Adding tensile forces between the grains, expectedly, does not make the problem simpler. Tensile forces can either be non reversible and lead to a brittle behaviour $[2,3]$, or be reversible, as for instance capillary forces in humid materials [4], and lead to a sticky cohesive behaviour [5]. In this contribution, we are interested in characterising the failure of sticky cohesive material.

We consider the failure of sticky cohesive squat heaps using discrete numerical simulations in two dimensions (2D). The onset of the failure, its orientation, and its evolution are presumably all dependent on the cohesive properties of the contacts, the frictional properties of the material, the shape of the system and the details of its initial state $[6,7]$. Disclosing the particulars of this dependence requires a dedicated comprehensive study. In this contribution, we are merely interested in exploring quantities that can be easily measured in numerical experiments, and which reveal the characteristics of the failure, namely onset and orientation. We track the moment of the failure and derive its orientation simply by distinguishing the grains regarding a displacement threshold.

The algorithm and numerical set-up are first presented in section 2. We explore the behaviour of one particular individual simulation in section 3 . We then quantify the reproducibility of the features discussed above by analysing the behaviour of 10 additional independent simulations in section 4 .

*e-mail: lydie.staron@upmc.fr

A video is available at https://doi.org/10.48448/9gvr-9f17
Table 1. Simulations performed

\begin{tabular}{lcccl}
\hline $\begin{array}{l}\text { No. of } \\
\text { grains }\end{array}$ & $\begin{array}{c}\text { Initial height } \\
H_{0}\end{array}$ & $B_{\text {ond }}$ & $\mu$ & $\begin{array}{l}\text { No. of } \\
\text { runs }\end{array}$ \\
\hline 5572 & $45 \mathrm{~d}$ & 30 & 0.2 & 11 \\
\hline
\end{tabular}

\section{Numerical experiments}

The granular systems were simulated applying a Contact Dynamics algorithm in two dimensions [8, 9]. A system in its initial state is shown in Figure 1. It is made of 5572 circular grains deposited under gravity, and left to fall onto a horizontal plane made rough by gluing grains on it. The roughness diameter is the same as the mean diameter of the free grains.

The diameter of the free grains is chosen randomly in the interval $[0.004 m ; 0.006 m]$, so that their mean diameter is $d=0.005 \mathrm{~m}$. The initiation of the random function changes the sequence of diameters ascribed to each grain, thereby allowing the generation of entirely different, independent initial states with identical number of grains and macroscopic dimensions.

The contacts are made cohesive through the introduction of an adhesive force limit depending on the weight of the grains and the $B_{\text {ond }}$ number [7, 10]:

$$
F_{\text {adh }}=-B_{\text {ond }} m_{i j} g,
$$

with $m_{i j}=2 \times\left(1 / m_{i}+1 / m_{j}\right)^{-1}$, and $i$ and $j$ are the two grains involved. The value of the Bond number is set to $B_{\text {ond }}=30$, and is not varied. The grains also interact at contact through Coulombic friction, involving the coefficient of friction $\mu$, set to $\mu=0.2$ and not varied in this paper. The dimensions of the granular step are: initial heigh $H_{0}=0.226 \mathrm{~m} \simeq 45 d$, with a horizontal extension $R_{0}=0.6 m \simeq 120 d$. This combination of height and value of $B_{\text {ond }}$ number coincides with an unstable state [7]. 
3 Failure of one example step: $\mu=0.2$ and $B_{\text {ond }}=30$

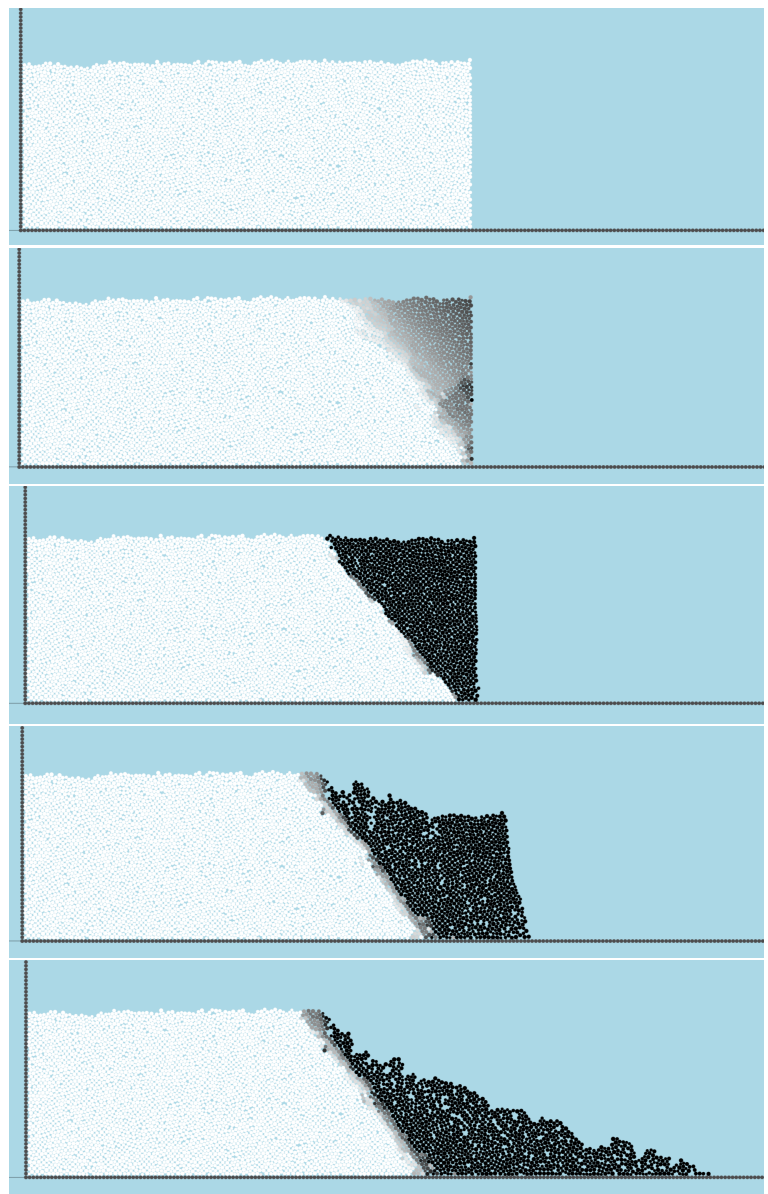

Figure 1. Successive snapshots of the failure of a cohesive granular step. Black colour coincides with a cumulative displacement $D>r_{t h}$, while white colour with a cumulative displacement $d<r_{t h} / 2$, with $r_{t h}=d / 2$. The grey colour scale is linear in this interval. Time shown are, from top to bottom, $t / T_{0}=0,0.2,0.39,1.32$ and $t / T_{0}=\infty, T_{0}=\sqrt{H_{0} / g}$. ( $B_{\text {ond }}=30$ and $\mu=0.2$ ).

\subsection{Identifying the failure}

The failure presumably occurs when the number of contacts opening as a result of tensile forces is sufficient and consistently located so as to allow a crack to propagate and a shear band to form as a result. We have not investigated the details of this early process that probably obeys a complex (and interesting) organisation. Instead, we focus on the cumulative displacement of the grains, which will start moving when a fracture opens and a shear band forms, with one side of the granular packing remaining static, and the other side breaking away. Instead of tracking the complex genesis of the fracture phenomena, we identify the failure through the emergence of the shearing zone, where grains will have, for a short time (before all broken material runs away), a bounded cumulative displacement.

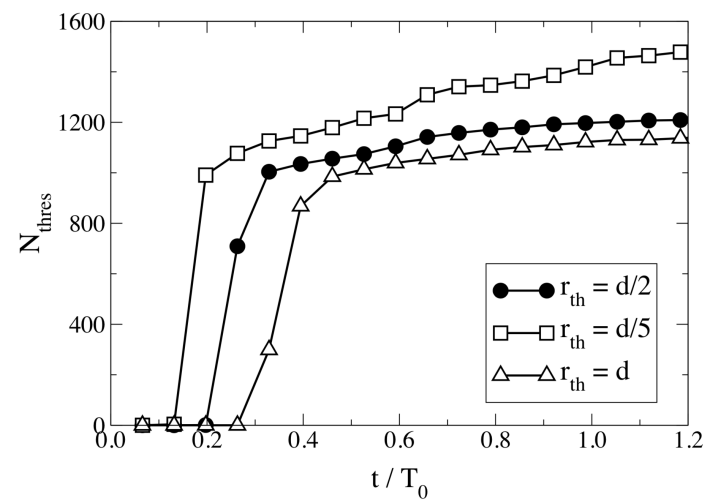

Figure 2. Number of grains whose cumulative displacement exceeds the threshold $r_{t h}$ as a function of the normalised time $t / T_{0}\left(T_{0}=\sqrt{H_{0} / g}\right)$ during the first moments of the failure. The evolution is shown for three values of the threshold: $r_{t h}=d / 2$, $d / 5$ and $r_{t h}=d$. The evolution retains a sharp-step shape in all cases, but the occurrence of the failure changes in time. $\left(B_{\text {ond }}=\right.$ 30 and $\mu=0.2$ ).

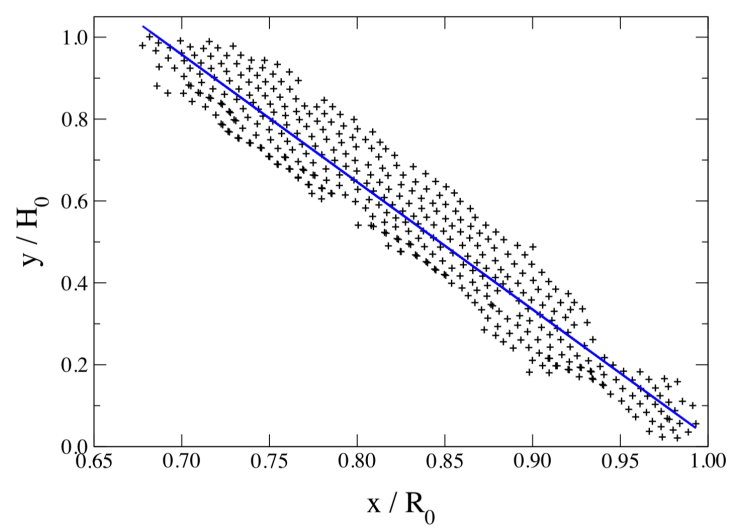

Figure 3. Position of the grains (normalised by the initial dimensions $R_{0}$ and $H_{0}$ ) whose cumulative displacement is comprised in $\left[r_{t h} / 2: r_{t h}\right]$, with $r_{t h}=d / 2$, between $t / T_{0}=0.2$ and $t / T_{0}=0.33$; the slope of the linear regression gives a failure angle (with the horizontal) $\psi=49.5 \mathrm{deg}$.

Therefor, we chose a displacement threshold $r_{t h}=d / 2$, and count the number $N_{t h}$ of grains with a cumulative displacement $D$ such that $D>r_{t h}$ to identify the grains which have started moving significantly. The evolution of $N_{t h}$ in the course of time is thus indicative of the occurrence of any event that induce grains motion. The results are displayed Figure 2 for the first instants of the failure. We observe a sharp evolution, bringing suddenly from zero to 1000 the number of grains having moved, after which it rapidly saturates and reaches a plateau, thus betraying a major yet short event. We define the characteristic time $T_{0}=\sqrt{H_{0} / g}$. Identifying the sharp increase with the formation of a shear band, we locate the shear to take place between times $t / T_{0}=0.2$ and $t / T_{0}=0.33$.

Two other values for $r_{t h}$ and the corresponding $N_{t h}$ are also displayed in Figure 2, showing the variations incurred when changing the displacement threshold. 


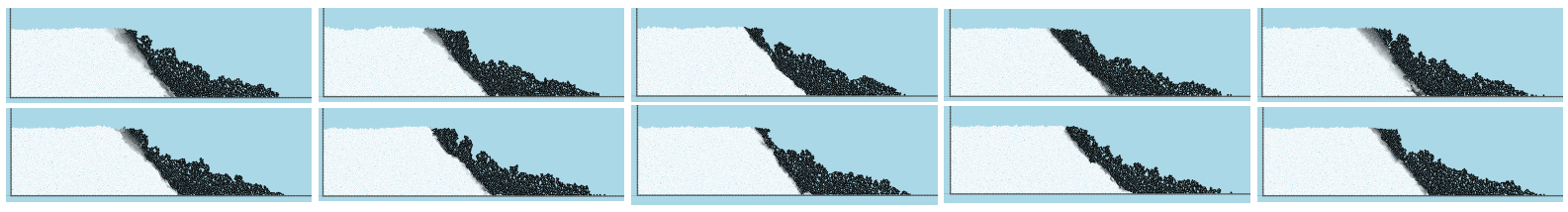

Figure 4. Final state and final deposit achieved after failure by ten independent granular steps with the same physical properties, but a different granular initial structure. All have a similar shape but are different and unique.

\subsection{Tracking down the shear band}

The identification of the instant of the failure is dependent on the displacement threshold we chose to measure the number of grains $N_{\text {thres }}$ displaced by the fracture. Two other solutions for the same breakage event are displayed in Figure 2: in addition to the threshold $r_{t h}=d / 2$, the number of grains $N_{\text {thres }}$ counted for a smaller threshold $r_{t h}=d / 5$, and a larger one $r_{t h}=d$, are shown. The evolution has the same general appearance in all three cases, but is shifted following the time axis: larger threshold, meaning larger displacements, are expectedly needing more time to reveal the failure.

In the absence of an unambiguous criteria to identify the instant (or time interval) of the failure, we chose the two points of the sharpest part of the evolution of $N_{\text {thres }}$ to define the time interval during which the shear band forms. For a threshold $r_{t h}=d / 2$, this interval is comprised between $t / T_{0}=0.2$ and $t / T_{0}=0.33$.

Focussing on this time interval, we track the grains having a cumulative displacement $D \geq r_{t h} / 2=d / 4$, keeping out those who have barely moved yet, namely, whose motion is induced in a second stage by erosion, and the grains having a cumulative displacement $D \leq r_{t h}=d / 2$, thus keeping out those who are moving away from the failure zone, or who are falling freely near the edges of the system. The position of these grains is plotted in Figure 3. They define a recognisable shear band, whose pattern gives an estimate of the failure direction $\psi$; a straightforward linear regression gives $\tan (\psi)=1.173$, namely $\psi \simeq 49.5 \mathrm{deg}$.

The choice of the threshold value for the grains displacement may affect this analysis. Depending on this value, the failure will be identified earlier or later, may involve a shear band thinner or thicker; it is not clear how much these details will weight in the determination of the failure angle $\psi$. We have checked that the main features were actually poorly dependent on the choice of $r_{t h}$, although only a systematic study can confirm this feeling.

There is however a greater source of uncertainty, which is the sensitivity of the failure to the details of the granular organisation: grain size distribution, contact network topology, tensile force pattern, all small-scale features whose role is difficult to assess. At this stage, we can simply give a measure of this sensitivity to the granular organisation by performing a series of similar step failure simulations, changing only the details of the granular packing.

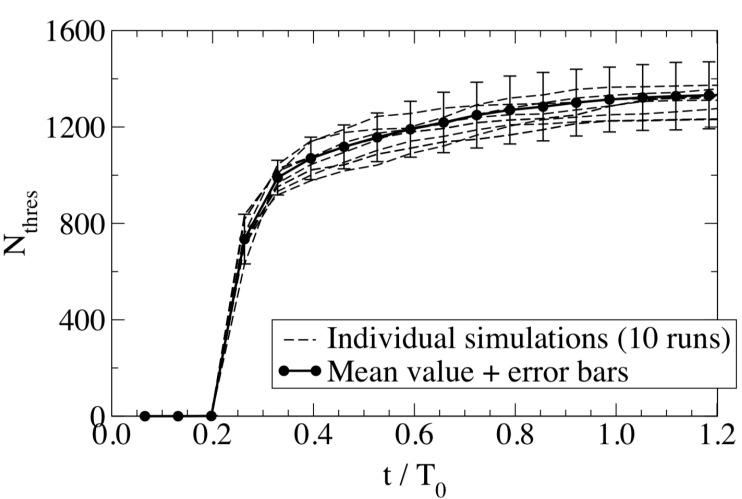

Figure 5. Number of grains whose cumulative displacement exceeds the threshold $r_{t h}$ as a function of the normalised time $t / T_{0}$ during the first moments of the failure. The evolution is shown for $r_{t h}=d / 2$ and for the 10 independent additional simulations, together with the mean evolution, and the corresponding error bars $\left(B_{\text {ond }}=30\right.$ and $\left.\mu=0.2\right)$.

\section{Robustness}

In order to estimate the error bar associated to the measures presented above, and to have a general idea of the reproducibility of the failure phenomena in a cohesive granular step, we generate 10 other independent similar systems. Therefor, we create granular packings with the exact same number of grains (5572 grains), the exact same material characteristics, i.e. the same contact properties (friction $\mu=0.2$ and cohesion $B_{\text {ond }}=30$ ). They differ only by the geometrical arrangements of the grains. This is achieved by randomly assigning grains diameters, following the procedure explained in section 2. Each packing is then left to fail and collapse under gravity, and the characteristics of the failure are analysed.

The analysis is performed using the same displacement threshold as used in the one exemple case examined in section 3, namely $r_{t h}=d / 2$. Figure 5 show the evolution in time of the number of grains $N_{t h}$ whose cumulative displacement has overcome $r_{t h}$, for each of the 10 additional simulations, and showing the mean evolution with corresponding error bars (computed as twice the interval between the maximum and minimum values centred on the mean). We observe that all failures follow the same general shape after the collapse has occurred. Most importantly, we observe for all 10 simulations that the fracture of the packing happens in the same time interval and is initiated at the same instant (namely $t / T_{0}=0.2$ ). We can thus conclude that the response in time to gravity of the gran- 


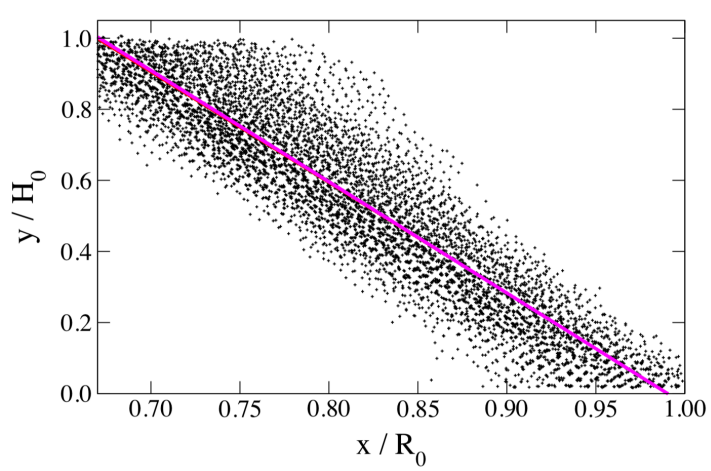

Figure 6. Position of the grains involved at the onset of shear motion for the 10 independent additional simulations (detected with a displacement threshold $r_{t h}=d / 2$ ) and corresponding linear regression with a slope suggesting a failure angle of 49.7 deg.

ular piles with $B_{\text {ond }}=30$ and $\mu=0.2$ is a robust feature and is fairly reproducible (although 10 remains a modest figure statistics-wise).

Having established, for the 10 additional simulations, that the failure occurs between $t / T_{0}=0.2$ and $t / T_{0}=0.33$, we can identify the position of the grains signalling the formation of a shear band as done in subsection 3.2. Figure 6 shows these positions superimposed on the same graph, and forming a "virtual" super shear band made of all 10 independent shear events. Each failure, analysed individually, allows for a linear regression with a correlation coefficient of typically 0.94 . The simulation series gives failure slopes ranging from $47.2 \mathrm{deg}$ to $51.1 \mathrm{deg}$, with a mean value of $49.7 \mathrm{deg}$.

Finally, the most erratic feature of the granular failure might be the profile of the final deposit, as suggested by Figure 4 and plotted in Figure 7. Although they all retain a common shape, namely a slightly concave slope of about $19.5 \mathrm{deg}$, the drawing of the profiles shows many variations, involving blocks of different sizes, from smooth decline to sharp break-away scar. The study of the flow following the failure and of the characteristics of the deposit are however beyond the scope of this study, but are the subject of a forthcoming work.

\section{Conclusion}

In this contribution, the failure of two dimensional granular steps was simulated using a Contact Dynamics algorithm. Considering one single set of values for the adhesive forces and the contact friction (namely $B_{\text {ond }}=30$ and $\mu=0.2$ ), we track the signature of the failure in the cumulative displacement of the grains. The introduction of a displacement threshold allows for the identification of the onset of a rapid failure and the formation of the corresponding shear band. The latter gives access to an estimation of the failure orientation.

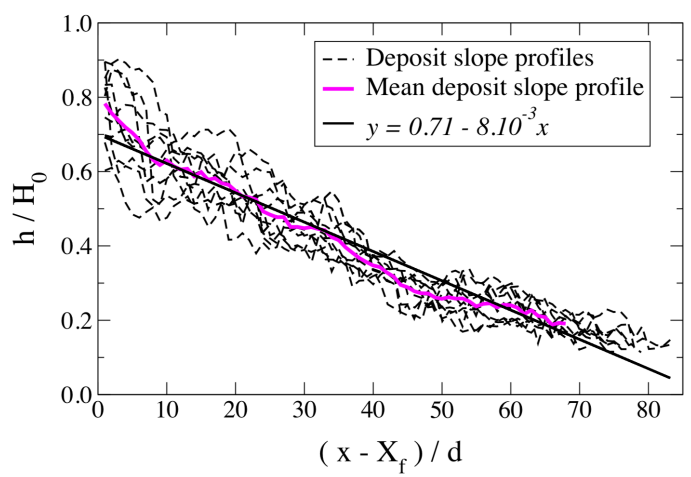

Figure 7. Final deposit slope profiles for the 10 simulations, together with the corresponding mean profile and the linear regression. All profiles are shifted to start at the point of the deposit $X_{f}$ where grains have a final cumulative displacement $\geq 2.5 d$, and are renormalised using the initial height $H_{0}$ and the mean grain diameter $d$.

The robustness of the measure thus performed is assessed by carrying out a series of 10 additional simulations, giving an estimate of the error bars, and showing a fairly reproducible behaviour. Exploring the dependences on the friction $\mu$ and on the cohesive forces through the $B_{\text {ond }}$ number is the logical following of this work.

This work is part of the COPRINT project supported by the ANR grant ANR-17-CE08-0017.

\section{References}

[1] S. Courrech Du Pont, P. Gondret, B. Perrin, M. Rabaud, EPL 61, 492 (2003)

[2] D. Bonamy, E. Bouchaud, Phys. Rep. 498,1-44 (2011)

[3] V.J. Langlois, A. Quiquerez, P. Allemand, J. Geophys. Res. 120,1866-1880 (2015)

[4] V. Richefeu, M.S. El Youssoufi, F. Radjai, Phys. Rev. E 73, 051304 (2006)

[5] A. Gans, O. Pouliquen, M. Nicolas, Phys. Rev. E 101, 032904 (2020)

[6] R.M. Nedderman, Statics and kinematics of granular materials, Cambridge University Press (1992)

[7] A . Abramian, L. Staron, P.Y. Lagrée, J. Rheol. 64, 1227-1235 (2020)

[8] J.-J. Moreau, Eur. J. Mech. A Solids 13, 93-114 (1994)

[9] F. Radjai, V. Richefeu, Mech. Mater. 41, 715-728 (2009)

[10] P.G. Rognon, J.-N. Roux, M. Naaïm, F. Chevoir, J. Fluid Mech 596, 21-47 (2008) 